\title{
AlertUFBA: um portal para apoiar situações de crise e emergência no contexto universitário
}

\author{
Ana Íris Santos ${ }^{1}$, Vaninha Vieira ${ }^{1}$, Maria Clara Pestana $^{1}$, Ailton Ribeiro ${ }^{1}$ \\ ${ }^{1}$ Universidade Federal da Bahia - (UFBA) \\ Salvador - BA - Brasil \\ \{iris.carvalho, vaninha, mpestana, ailton.ribeiro\}@ufba.br
}

\begin{abstract}
Effective management in predicting occurrences preserve a community constantly informed about the risks of disaster in its surroundings, thus preventing an affected population from being harmed. This research presents AlertUFBA, an online portal to support risky and emergency situation in the context of UFBA's university campus. The management transmits and receives data from external sources and the affected audience to contribute to the institution's decision-making.
\end{abstract}

Key-words: emergency, crisis, communication.

Resumo. Para manter uma comunidade constantemente informada sobre possiveis riscos de desastre em seu entorno, é necessário que exista uma gestão eficaz na previsão de ocorrências, e assim evitar que a população afetada seja prejudicada. Essa pesquisa apresenta o AlertUFBA, um portal online para apoiar situações de risco e emergência no contexto de campus universitário da UFBA. A gestão será feita através da transmissão e recepção de dados advindos de fontes oficiais externas e do público alvo afetado que possam contribuir para a tomada de decisão pelos frequentadores da instituição.

Palavras-chave: emergência, crise, comunicação.

\section{Introdução}

A comunicação tem papel fundamental no compartilhamento de informações e transmissão de mensagem em tempo real. Já em situações de crise, a comunicação se torna vital, pois é neste momento em que a administração de instituições precisam estar atentos para transmitir a mensagem que for necessária para as partes interessadas.

Os sistemas que enviam alertas de emergência, são chamados de Sistemas de Alerta Antecipado, (EWS, em inglês)[Lobo 2019]. Esses sistemas geralmente usam o Common Alerting Protocol (CAP). O formato CAP é compatível com técnicas emergentes, como serviços da Web, bem como formatos existentes, incluindo a codificação de mensagem de área específica (SAME) usada para o rádio meteorológico da Administração Nacional Oceânica e Atmosférica dos Estados Unidos (NOAA) e o sistema de alerta de emergência (EAS) [Standard Oasis 2010].

Segundo informações do IBGE (2018) após análise dos dados do Censo Demográfico de 2010, o Brasil tem mais de 8 milhões de pessoas que vivem em áreas com risco de enchentes e deslizamentos de terra, sendo a maioria delas moradoras da cidade 
de Salvador (1,2 milhão) [IBGE 2018]. Tendo em vista tais ocorrências, o presente desenho de pesquisa apresenta uma solução centrada em um portal, AlertUFBA, para apoiar a gestão de crise e emergência sujeitos a afetar a comunidade do campus universitário da UFBA. Este serviço é baseado no recebimento de informações antecipadas sobre riscos e envio de alertas para anunciar medidas para mitigar danos a saúde da comunidade, conservando as atividades de ensino e pesquisa e o patrimônio.

\section{Revisão de Literatura}

Com a globalização é possível observar a evolução rápida da Internet e os recursos computacionais que disponibilizam para a sociedade uma gama de informações na WEB. Essas informações podem ser úteis no apoio a gestão de crise e emergência que envolve o processo de ocorrência de desastres.

Segundo documento de gestão de risco de desastre do governo de Santa Catarina [Governo de Santa Catarina 2018], o glossário de Defesa Civil de 2007 define que o risco de desastre é a relação existente entre a probabilidade de que uma ameaça de evento adverso ou acidente determinado se concretize. O documento define desastre como “(...) o resultado de eventos adversos, naturais ou provocados pelo homem, sobre um cenário vulnerável, causando grave perturbação ao funcionamento de uma comunidade ou sociedade; envolvendo extensivas perdas e danos humanos, materiais, econômicos ou ambientais, (...)". O presente trabalho considera a gestão de crise e emergência como ações que visam evitar a ocorrência de um desastre.

A FEMA[FEMA 2015], agência responsável pela comunicação de desastres nos Estados Unidos disponibiliza um serviço chamado Emergency Alert System (EAS) que envia mensagens broadcast via cabo, satélite e outros meios, informando os cidadãos sobre incidente em dado local, e funciona mesmo quando os meios de alerta público estão indisponíveis. Para funcionar em vários dispositivos, exige a implementação de um protocolo de comunicação específico, para uso em TVs, rádio, satélite e Internet. Os Estados Unidos, adotou formalmente o padrão CAP e está sendo usado como a tecnologia básica para o abrangente Sistema Integrado de Alerta e Alerta Público (IPAWS) [Sean Matthews 2016].

No contexto universitário, a Universidade do Alabama possui um Plano de Operações de Emergência que incorpora protocolos do Sistema Nacional de Gerenciamento de Incidentes (NIMS) para seus steakholders. Ela possui um abrangente gerenciamento de emergências, inclusive possui certificação pela Agência de Credenciamento de Gerenciamento de Emergência (EMAP) e também é uma Universidade StormReady certificada pelo Serviço Nacional de Meteorologia [Sean Matthews 2016]. Além disso, a universidade disponibiliza no seu site recurso para estudantes, funcionários e visitantes para aprimorar sua preparação para emergências e ainda é possível encontrar alertas sobre o tempo.

A universidade americana Stanford University utiliza de duas estratégias de notificação em massa para comunicar informações sensíveis ao tempo durante um evento de emergência que afeta o campus. Por isso, a depender da natureza do incidente será usado um desses dois métodos para alertar a comunidade do campus: O AlertSu - sistema de notificação em massa que envia mensagens via SMS, e-mail ou telefone para membros da comunidade de Stanford. O sistema de alerta externo é composto por sete sirenes 
posicionadas em todo o campus principal que emitem tons de alerta e instruções verbais destinadas a alcançar aqueles que estão ao ar livre.

As mensagens de alerta podem ser enviadas para a comunidade de Stanford via mensagem de texto, e-mail e, em casos extremos, o sistema de sirenes externas e os altofalantes VoIP [University 2016]. O AlertSu envia mensagem, para estudantes, professores e funcionários. A comunidade externa pode acompanhar as informações no site ${ }^{1}$ da universidade. As informações de contato precisam estar sempre atualizadas, para estudantes é feita a integração com o sistema Axess, enquanto professores e funcionários, os dados são integrados ao StanfordYou.

No Brasil, em Santa Catarina, na cidade de Blumenau, o AlertaBLU é um sistema de monitoramento e alerta de chuvas intensas que tem como objetivo prever, monitorar e alertar a comunidade sobre condições meteorológicas que possam causar transtornos e prejuízos em tempo real. Pode-se compreender o AlertaBLU como um procedimento através do qual se recolhe e analisa informações sobre ameaças, a fim de alertar a população vulnerável antes de um impacto [Ludwig 2018].

\section{Justificativa}

Lidar com crises requer um comportamento de precaução, pois os processos de gestão começam antes mesmo da crise se consolidar. A intenção é que a organização esteja um passo à frente, no sentido de saber o que pode acontecer, em vez de aguardar o caso vir a público e logo depois tomar medidas [dos Santos 2016]. Antecipar significa executar medidas como a evacuação de áreas de risco que pode ser decisiva para evitar vítimas e danos socioeconômicos [Saito 2013]. Por exemplo, na ocorrência de chuvas, quanto mais impermeabilizada a área, mais sujeita a inundações e alagamentos estará a cidade, Salvador, assim como outras grandes cidades do Brasil sofre periodicamente o problema das enchentes, com alagamentos e deslizamentos do terreno por causa das chuvas [Sanchez 2014].

No ano de 2019, a UFBA teve ocorrências de inundações por conta das fortes chuvas que caíram em Salvador, em que aulas foram suspensas. Em 2015, a UFBA recomendou flexibilização nos dias 27 e 28 de abril das atividades por conta das chuvas. Além da ocorrência de chuvas, podem existir outras situações prejudiciais a comunidade acadêmica. Ocorre que nem sempre as universidades conseguem ter uma preparação ou resposta imediata a estas situações adversas, tornando-se um local de vulnerabilidade, então ter uma ferramenta de apoio a gestão de ocorrências severas é de suma importância, principalmente quando não há um setor específico e nem procedimentos documentados para a tomada de decisão durante uma situação de crise.

\section{Proposta}

A UFBA ainda carece de ferramentas que apoiem a comunicação em situações de crise e emergência. Sabe-se que as atuais ferramentas de comunicação utilizada na UFBA são: o site oficial e as redes sociais dos Santos 2016]. Segundo a Assessoria de Comunicação Institucional da UFBA (Ascom), a Internet é a principal mídia para circulação de tudo que é produzido pelo setor e revela oportunidades de melhorias no tocante ao ambiente

\footnotetext{
${ }^{1}$ http://emergency.stanford.edu
} 
on-line. A Ascom, tem atuado principalmente com o público externo. Em situações que envolvam situações com a comunidade interna, a nota é feita em conjunto com a reitoria. Como por exemplo, a nota que saiu no dia 26/11/2019 após fortes chuvas na cidade com vários pontos de alagamentos no campus, sendo necessário o cancelamento das atividades.

Diante das ocorrências estudadas, a presente pesquisa propõe construir e avaliar uma solução centrada no portal AlertUFBA com o objetivo de disponibilizar para a comunidade da UFBA alertas e informações para que os estudantes, professores e funcionários possam evitar estar diante de um desastre emergente. A visão geral da proposta está apresentada na Figura 01. Essa visão sugere uma prévia de como seria a arquitetura da solução a ser desenvolvida posteriormente.

Os dados podem vir de duas maneiras, a primeira seria de fontes oficiais externas, e a segunda seria da própria população, ou seja, os frequentadores do campus da UFBA. Para o estudo de caso inicialmente estudado, serão utilizados dados do site do INMET, que serão armazenados em um servidor local, que possa servir como apoio a ocorrência de desastres próximos a Universidade. Outras fontes de dados sobre ocorrências nas mediações do campus também podem ser integradas a este serviço posteriormente, no decorrer do andamento da pesquisa. A outra fonte de dados externas são as informações advindas da população do campus. O servidor também será alimentado por opiniões e sugestões do público quanto as situações de riscos sujeitas a ocorrer no campus da UFBA ou com seus frequentadores.

As informações contidas neste servidor funcionaria como um modelo para gestores se basearem ao tomar decisões que poderá alertar a comunidade que será afetada. Conforme decisão que o órgão deverá tomar diante da situação de risco, a comunidade será alertada de como deverá agir. Este alerta será enviado através dos canais de comunicação como telegram, e-mail, site da UFBA, telas digitais e redes sociais. E os estudantes, técnicos e servidores que estão a caminho da instituição poderão fornecer informações de como está o trajeto ao se deslocar até a universidade.

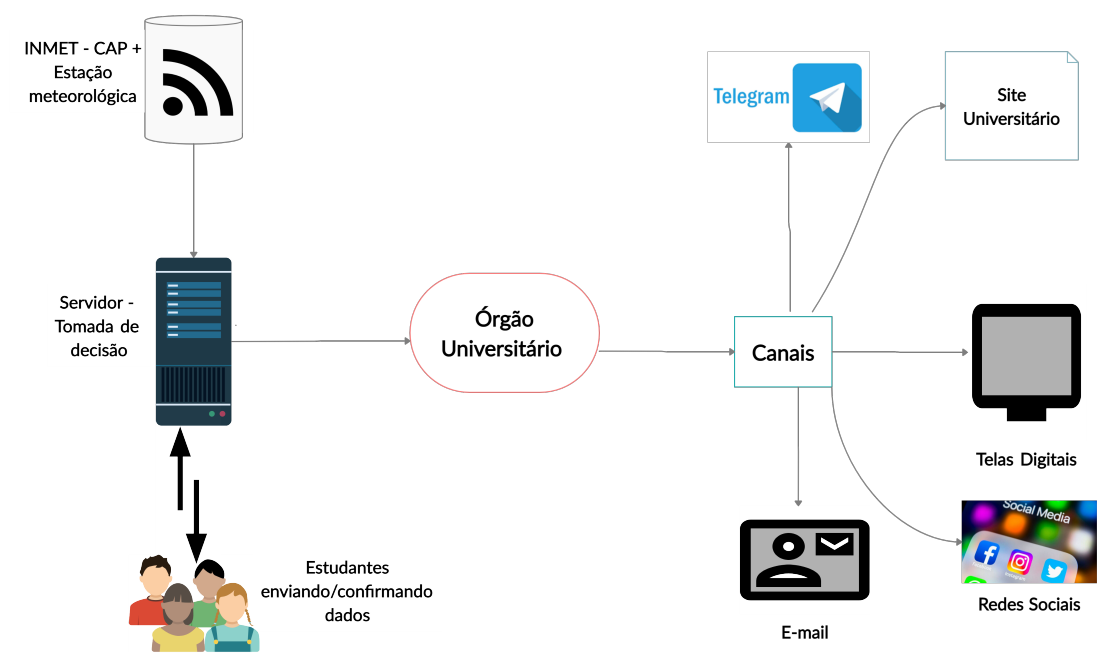

Figura 1. Visão geral da proposta: prévia da arquitetura da solução. 


\section{Metodologia}

O trabalho será desenvolvido através de pesquisa exploratória, entrevistas e consultas ao público do campus da UFBA, prototipação da solução e avaliação.

\subsection{Pesquisa Exploratória}

Com o intuito de delinear o contexto e as ocorrências de desastres na UFBA, foram realizadas 3 entrevistas. A primeira foi na Superintendência de Tecnologia da Informação, onde obtivemos respostas e posicionamento de como ocorreu a comunicação e como foi os procedimentos adotados. O caso descrito foi sobre uma inundação que fez com que o equipamento da unidade queimasse na década de 80. A entrevistada relatou que não estavam preparados para o ocorrido e a comunicação ocorreu de forma improvisada.

A segunda entrevista foi no Núcleo de Apoio a Inclusão de Aluno com Necessidades Especiais, onde foi obtida a informação de que a unidade utiliza como meio de comunicação não-oficial o Whatsapp, e que a única dificuldade que tem é em sempre estar fazendo a descrição da imagem para os deficientes. Isso sugere a falta de preparação e estrutura para atender pessoas com deficiência que estejam no campus durante uma situação de desastre.

A terceira entrevista foi realizada na Assessoria de Comunicação Institucional onde foram feitas perguntas sobre os meios de comunicação em situação de crise. Através das respostas da entrevista, conclui-se que os meios de comunicação utilizados são portais da Internet e redes sociais. $\mathrm{O}$ setor vem passando por constantes reestruturações para atender a comunidade. O setor da universidade responsável por atender as demandas de situação de emergência é a Reitoria, que por concentrar uma alta demanda de processos, pode enfrentar dificuldades para gerir essas situações. Há a necessidade de se preparar para situações de crise, no sentido de ensaiar cenários que evitem danos as pessoas envolvidas e qualificar periodicamente os funcionários para este tipo de ocorrência.

\subsection{Prototipação}

A proposta apresentada na Figura 01 é uma prévia da arquitetura a ser desenvolvida na presente pesquisa que é centrada no portal AlertUFBA. Porém, é preciso estabelecer como essa integração entre o portal e as fontes externas serão integrados, assim como, as entradas de dados prestadas pelo público.

O primeiro caso estudado abordou a ocorrência de chuvas, através do banco de dados do INMET, que funciona como uma fonte de dados oficial do governo. O INMET possibilita mapear dados meteorológicos da cidade de Salvador, mais especificamente, uma estação meteorológica automática localizada no bairro de Ondina, região onde um campus da UFBA está situado, sendo possível haver alertas quanto ao desastres provenientes de fortes chuvas. Também serão estudados formas de integrar o portal com outras fontes oficiais do governo, sistemas acadêmicos e soluções desenvolvidas pelos estudantes e pesquisadores da instituição.

\subsection{Avaliação}

Afim de obter maiores detalhes sobre as ocorrências e a viabilidade do portal AlertUFBA, será feito uma nova pesquisa com um questionário utilizando o modelo TAM (Modelo de Aceitação da Tecnologia) que consiste em explicar e prever a aceitação de tecnologia (AlertUFBA) pelos frequentadores do campus. 


\section{Conclusão}

Uma comunicação de crise bem gerenciada em uma instituição de ensino superior durante uma emergência pode evitar grandes transtornos. Diversos atores (estudantes, professores, diretores, reitoria, segurança patrimonial, visitantes, defesa civil, entre outros) estão envolvidos em uma situação de crise na universidade. A colaboração entre estes atores para uma comunicação eficaz é um desafio antes, durante e após uma crise. A presente pesquisa propõe o AlertUFBA, um portal online para alertar as pessoas da UFBA sobre situações de emergência sujeitas a ocasionar desastres. Com esse portal as partes interessadas serão alertadas diante daquela ocorrência, podendo assim ser evitado danos a vida e ao patrimônio.

\section{Referências}

[dos Santos 2016] dos Santos, J. A. L. (2016). Gestão de Crise e Comunicação Organizacional: Uma Análise do Problema de Segurança na Universidade Federal da Bahia. Monografia do Repositório da Universidade Federal da Bahia.

[FEMA 2015] FEMA (2015). Federal emergency management agency: Disaster emergency communications. Disponível em: https://www.fema.gov/ disaster-emergency-communications Acesso em: 23 de março de 2021.

[Governo de Santa Catarina 2018] Governo de Santa Catarina (2018). GestÃo de risco de desastres. Disponível em: encurtador.com. br/EKLP1. Acesso em: 23 de março de 2021.

[IBGE 2018] IBGE (2018). População em áreas de risco no brasil. Disponível em: https : //www.ibge.gov.br/apps/populacaoareasderisco. Acesso em: 11 novembro 2019.

[Lobo 2019] Lobo, J. S. e Vieira, V. (2019). Mypersonalews: Uma abordagem colaborativa para alertar surdos, cegos e analfabetos sobre desastres. SBSC, page 1.

[Ludwig 2018] Ludwig, L. (2018). As tecnologias da informação e comunicação na gestão dos riscos de desastres: o caso do alertablu em blumenau/sc. r. tecnol. soc. v. 14, n. 31, p. 92-109.

[Saito 2013] Saito, S. M. e de Souza, D. O. (2013). Sistema de Monitoramento e Alerta de Desastres Naturais: praticas e desafio, chapter 9, page 208. Eventos Extremos no Rio Grande do Sul: inundações e movimentos de massa.

[Sanchez 2014] Sanchez, A. S. (2014). Aproveitamento de Águas pluviais na escola politécnica da ufba. Dissertação de Mestrado do Repositório da Universidade Federal da Bahia.

[Sean Matthews 2016] Sean Matthews (2016). Common alerting protocol (cap) for higher education institutions. Disponível em: encurtador.com.br/blwAX. Acesso em: 11 novembro 2019.

[Standard Oasis 2010] Standard Oasis (2010). Common alerting protocol. Disponível em: http://docs.oasis-open.org/emergency/cap/v1.2/CAP-v1. 2-os.html Acesso em: 23 de março de 2021.

[University 2016] University, S. (2016). University emergency plan. Disponível em: encurtador.com.br/tQX34 Acesso em 23 de março de 2021. 